\title{
Equilibrium swelling properties of polyampholytic hydrogels
}

\author{
Anthony E. English \\ Department of Nuclear Engineering and Harvard-MIT Division of Health Sciences and Technology, \\ Massachusetts Institute of Technology, Cambridge, Massachusetts, 02139
}

Salvador Mafé, ${ }^{\text {a) }}$ José A. Manzanares, ${ }^{\text {a) }}$ Xiahong Yu, Alexander Yu. Grosberg, ${ }^{\text {b) }}$ and Toyoichi Tanaka

Department of Physics and Center for Materials Science and Engineering,

Massachusetts Institute of Technology, Cambridge, Massachusetts 02139

(Received 4 October 1995; accepted 16 February 1996)

\begin{abstract}
The role of counter ions and ion dissociation in establishing the equilibrium swelling of balanced and unbalanced polyampholytic hydrogels has been investigated experimentally and theoretically. The swelling dependence on both the net charge offset and the external bath salt concentration has been examined using an acrylamide based polyampholytic hydrogels. By careful consideration of the swelling kinetics, we illustrate the effects of ion dissociation equilibria and counter ion shielding in polyampholytic hydrogels near their balance point where both polyelectrolyte and polyampholyte effects are present. The theory considers a Flory type swelling model where the Coulombic interactions between fixed ions in the hydrogel resemble those of an ionic solid with a Debye screening factor. Theoretical predictions from this model are in qualitative agreement with our experimental results. (C) 1996 American Institute of Physics. [S0021-9606(96)51119-6]
\end{abstract}

\section{INTRODUCTION}

Polyampholytic hydrogels are cross-linked polymer networks that are made up of both positive and negative monomeric groups with the possible inclusion of neutral monomeric spacers. Balanced polyampholytes have an equal number of positive and negative charges while unbalanced polyampholytes have a charge excess. The long range Coulombic interactions which determine the physical properties of polyampholytes are a challenge to both theoretical and experimental understanding.

In recent years, considerable effort has been devoted to understanding the single polyampholyte chain in very dilute solutions where counter ions do not screen the Coulombic potential. It has been shown in extensive studies by Kantor and $\operatorname{Kardar}^{1-4}$ and by Shakhnovich and Gutin ${ }^{5}$ and Dobrynin and Rubinstein ${ }^{6}$ that polyampholyte chains form a spherical globular structure when the overall net charge does not exceed $q_{0} \sqrt{N}$. When the net charge exceeds $q_{0} \sqrt{N}$ the polyampholyte forms an elongated globule. The globule density is determined by the overall attraction and is almost independent of the charge excess while the globule shape is governed primarily by the net charge.

A macroscopic polyampholytic hydrogel, however, is dramatically different. On length scales greater than a Debye length the distribution of charged monomers, counter ions, and co-ions maintains almost perfect charge neutrality. This creates a complicated set of interactions within the system. Unlike a plasma or electrolyte, several components contribute to the screening in a different manner. ${ }^{7}$ The charged monomers of both signs, counter ions and co-ions all have

\footnotetext{
a) On leave from: Department of Thermodynamics, University of Valencia, 46100 Burjasot, Spain.

${ }^{b)}$ On leave from: Institute of Chemical Physics, Russian Academy of Sciences, Moscow 117977, Russia.
}

different degrees of freedom to respond to local electric fields. In addition, acid and base ion dissociation equilibrium can have important effects on the polymer charge density.

Polyampholytes have been the subject of a number of experimental studies. Corpart et al. ${ }^{8}$ and Skouri et al. ${ }^{9}$ examined the aqueous solution properties of polyampholytes using viscometry and light scattering measurements. Baker et al. ${ }^{10}$ later examined balanced polyampholyte hydrogel swelling and proposed a semiquantitative model based on a Flory type theory with Debye-Hückel contributions. These experimental studies are in qualitative agreement with Higgs and Joanny's model. ${ }^{11}$ Balanced polyampholytes collapse at low salt concentrations and swell at high salt concentrations. With increasing charge offset, polyelectrolyte behavior is observed and swelling decreases with increasing salt concentration. For polyampholytes near their balance point, however, both effects can be important over a wide range of bath electrolyte concentrations. ${ }^{12,13}$

In this work we examine the swelling equilibria of polyampholytic hydrogels both experimentally and theoretically. The theory considers a Flory type swelling model for ionic hydrogels where the Coulombic interactions between the hydrogel fixed ions resemble those of an ionic solid with a Debye screening factor. Higgs and Joanny's theory was based on the assumption that all charges, both mobile and polymeric, contribute equally to the screening. One would expect that the polymer chain connectivity and monomer masses will significantly restrict the polyions compared to the mobile counter and co-ions. As a result, we consider the limit where the monomers are immobile and do not adjust to fluctuating fields or contribute to the screening. By careful consideration of the swelling kinetics, we illustrate the additional effects of ion dissociation equilibria using acrylamide based polyampholytic hydrogels near their balance point 
where both polyelectrolyte and polyampholyte effects are present.

\section{THEORETICAL MODEL}

Thermodynamic equilibrium is achieved when the chemical potential of each mobile species is equal in every phase. A balance of solvent chemical potentials is a statement of osmotic pressure or swelling equilibrium. Similarly, ionic equilibrium of mobile species is obtained when the electrochemical potentials within the hydrogel equal that in the external bath. For those species capable of binding to functional groups, the electrochemical potentials in the bound and unbound states must also be equal. The volumetric charge density will therefore be a complicated function of the swelling process and a model which considers both swelling and dissociation equilibrium must be constructed.

\section{A. Free energy model}

We identify four contributions to the free energy and assume they are additive,

$$
\Delta F=\Delta F_{M}+\Delta F_{\text {el }}+\Delta F_{\text {trans }}+\Delta F_{\text {Coulombic }},
$$

where $\Delta F_{M}, \Delta F_{\text {el }}, \Delta F_{\text {trans }}$, and $\Delta F_{\text {Coulombic }}$ represent the mixing, elastic, translational, and Coulombic contributions to the free energy, respectively.

Based on the Flory-Huggins lattice theory, ${ }^{14}$ the free energy of mixing between monomer and solvent molecules is

$$
\Delta F_{M}=k_{B} T \frac{V}{v_{\text {site }}}(1-\phi)[\ln (1-\phi)+\chi \phi],
$$

where $V$ is the hydrogel volume, $v_{\text {site }}$ the lattice site volume, $\phi$ the polymer volume fraction, $\chi$ the polymer solvent interaction parameter, $k_{B}$ is Boltzmann's constant, and $T$ the absolute temperature.

From the affine network model, ${ }^{14,15}$ the elastic contribution to the free energy is

$$
\Delta F_{\mathrm{el}}=\frac{3 k_{B} T}{2} \frac{V \phi}{N_{x} v_{\text {site }}}\left[\left(\frac{\phi_{o}}{\phi}\right)^{2 / 3}-1-\frac{1}{3} \ln \left(\frac{\phi_{o}}{\phi}\right)\right],
$$

where $N_{x}$ is the average number of monomers between crosslinks. The term $V \phi / N_{x} v_{\text {site }}$ is the effective number of chains in the network while $\phi_{o} / \phi$ is the swelling ratio. Hydrogel volumes and polymer volume fractions are related through

$$
\frac{V}{V_{0}}=\frac{\phi_{0}}{\phi}=\left(\frac{D}{D_{0}}\right)^{3},
$$

where $V_{0}, D_{0}$, and $\phi_{0}$ are the hydrogel volume, diameter, and polymer volume fraction in the reference state, respectively.

The translational component represents the ion mobility contribution to the free energy. For this term we consider the ideal gas contribution,

$$
\Delta F_{\text {ideal }}=k_{B} T \sum_{i} N_{i}\left\{\ln \left(\frac{N_{i}}{V}\right)^{-1}\right\},
$$

where $N_{i}$ is the total number of of a given mobile ion. The sum is over all mobile ion species such that

$$
C_{\text {mobile }} \equiv \sum_{i} \frac{N_{i}}{N_{A} V}
$$

is the total molar mobile ion concentration and $N_{A}$ is Avogadro's number. We assume that the excluded volume correction to $V$ in Eq. (5) is negligible. The Donnan equilibrium relations with the constraint of local charge neutrality lead to

$$
C_{\text {mobile }}=2 \sqrt{\left(\frac{N_{f^{+}}-N_{f^{-}}}{2 N_{A} V}\right)^{2}+C_{0}^{2}},
$$

where $N_{f^{+}}$and $N_{f^{-}}$are the total number of positive and negative fixed charges, respectively. If $C_{S}$ and $C_{\mathrm{H}^{+} \text {Bath }}$ represent the bath salt and hydrogen ion concentrations then $C_{0}=C_{S}+C_{\mathrm{H}^{+} \text {Bath }}$.

The Coulombic contribution to the free energy is

$$
\Delta F_{\text {Coulombic }}=\sum_{i} \mathrm{z}_{i} e N_{i} \phi_{D}+\Delta F_{\text {lattice }},
$$

where $\phi_{D}$ is the Donnan potential and $\Delta F_{\text {lattice }}$ represents the contribution due to the interaction between fixed charge groups. The lattice free energy, $\Delta F_{\text {lattice }}$, can be evaluated by considering the bound polymer charges as a quasilattice with screened Coulomb interactions. We now describe this term by considering a lattice model where every site is surrounded by $z$ nearest neighbors.

If only nearest-neighbor interactions are considered, the lowest energy configuration of a balanced polyampholyte $\left(N_{f^{+}}=N_{f^{-}}\right)$would be obtained when every charge has $z$ oppositely charged neighbors. The configuration energy would then be

$$
\Delta F_{\text {lattice }}=-\frac{z}{2}\left(N_{f+}+N_{f-}\right) \frac{e^{2}}{4 \pi \epsilon} \frac{\exp (-\kappa r)}{r},
$$

where $e$ is the electron charge, $\epsilon$ is the dielectric permittivity of the solvent, $r$ the nearest neighbor distance such that

$$
r \approx 2\left(\frac{3 V}{4 \pi\left(N_{f^{+}}+N_{f^{-}}\right)}\right)^{1 / 3},
$$

and

$$
\kappa=\sqrt{\frac{e^{2} N_{A} C_{\text {mobile }} 10^{3}}{\epsilon k_{B} T}}
$$

is the reciprocal Debye length in the hydrogel.

If some positive fixed charges are replaced by negative charges, the lowest energy would be obtained when every positive charge has the maximum number $z$ of oppositely charged nearest neighbors. This would give $z N_{f^{+}}$attractive interactions and $(z / 2)\left(N_{f^{+}}+N_{f^{-}}\right)-z N_{f^{+}}$repulsive interactions. The system free energy in this case would be

$$
\Delta F_{\text {lattice }}=\frac{z}{2} N_{f e} \frac{e^{2}}{4 \pi \epsilon} \frac{\exp (-\kappa r)}{r},
$$


where $N_{f e}=N_{f+}+N_{f-}-2\left|N_{f+}-N_{f-}\right|$ is the effective number of fixed charges giving rise to attractive interactions. The absolute value permits us to consider both excess positive or negative fixed charge.

The modification of Eq. (12) to include long range interactions involves replacing the number of nearest neighbors, $z$, by a parameter similar to the Madelung constant of ionic crystals. ${ }^{16}$ Retaining the Debye screening term gives us

$$
\Delta F_{\text {lattice }}=-\frac{A}{2} N_{f e} \frac{e^{2}}{4 \pi \epsilon} \frac{\exp (-\kappa r)}{r},
$$

where $A$ must be of the order of unity. ${ }^{16}$ Equation (13) represents the polyampholyte effect which is screened at high salt concentrations.

Although it has received serious criticism, ${ }^{17}$ the screened potential is common in the polyelectrolyte literature. ${ }^{18,19} \mathrm{We}$ expect the screened potential, however, contains the essentials of the screening effect and to be suitable for the study of balanced and slightly unbalanced polyampholytes.

\section{B. Swelling equilibrium}

Balance of solvent chemical potentials is generally expressed as

$$
\Delta \Pi_{\text {swelling }}=\frac{\mu_{s}^{\mathrm{gel}}-\mu_{s}^{\mathrm{bath}}}{\bar{v}_{w}}=0,
$$

where $\bar{v}_{w}$ is the solvent molar volume. Equating hydrogel and bath solvent chemical potentials gives us the swelling equilibrium condition

$$
\begin{aligned}
& -\frac{1}{\bar{v}_{\text {site }}}\left[\ln (1-\phi)+\phi+\chi \phi^{2}\right]+\frac{\phi_{0}}{N_{x} \bar{v}_{\text {site }}}\left[\frac{1}{2}\left(\frac{\phi}{\phi_{0}}\right)-\left(\frac{\phi}{\phi_{0}}\right)^{1 / 3}\right] \\
& +C_{\text {mobile }}-2 C_{0}-\frac{A}{2} \frac{N_{f e}}{N_{A} V} \frac{e^{2}}{12 \pi \epsilon k_{B} T} \frac{\exp (-\kappa r)}{r} \\
& \quad \times\left\{1+\kappa r-\frac{3 \kappa r}{2}\left[1+\left(\frac{2 N_{A} V C_{0}}{N_{f^{+}}-N_{f^{-}}}\right)^{2}\right]^{-1}\right\}=0,
\end{aligned}
$$

where $\bar{v}_{\text {site }} \equiv N_{A} v_{\text {site }}$ represents the molar volume of the lattice sites. The terms inside the curly brackets arise from the volume dependence of $\kappa$ and $r$.

\section{Dissociation equilibria}

The balance of chemical potentials for internal hydrogen and hydroxide ions in the free and bound states is equivalent to the chemical equilibrium of acidic and basic monomeric species,

$$
K_{a}=\frac{C_{A^{-}} C_{\mathrm{H}^{+}}}{C_{A \mathrm{H}}}
$$

and

$$
K_{b}=\frac{C_{B \mathrm{OH}} C_{\mathrm{H}^{+}}}{C_{B^{+}}} .
$$

The internal parameters $K_{a}$ and $K_{b}$ represent the acid and base dissociation constants, $C_{A^{-}}$and $C_{B^{+}}$the concentration of ionized acid and base groups, $C_{A H}$ and $C_{B \mathrm{OH}}$ the concentration of unionized acid and base groups, and $C_{\mathrm{H}^{+}}$and $C_{\mathrm{OH}^{+}}$the concentration of hydrogen and hydroxide ions. Localized hydrogen or hydroxide ion binding is an important consideration since it leads to a reduction in local charge repulsion and osmotic pressure in unbalanced polyampholytes.

When $\mathrm{CO}_{2}$ is dissolved in water there is a chemical interaction. Most of the dissolved $\mathrm{CO}_{2}$ becomes hydrated, in a way that is not completely understood, and a small fraction of it forms carbonic acid based on the sequence

$$
\mathrm{CO}_{2}+\mathrm{H}_{2} \mathrm{O} \rightarrow \mathrm{H}_{2} \mathrm{CO}_{3} \rightarrow \mathrm{H}^{+}+\mathrm{HCO}_{3}^{-} .
$$

The acid dissociation constant is

$$
K_{c}=\frac{C_{\mathrm{H}^{+}} C_{\mathrm{HCO}_{3}^{-}}}{C_{\mathrm{H}_{2} \mathrm{CO}_{3}}} .
$$

The effect of the bicarbonate ion is important because its concentration is 250 times larger than the hydroxide ion if base is not added. The effect of the bicarbonate ion can be avoided by running the experiment under a nitrogen or argon atmosphere.

Solving for the molar concentration of hydrogen ions, $C_{\mathrm{H}^{+}}$, inside the hydrogel gives us the fourth order algebraic equation

$$
\begin{aligned}
C_{H^{+}}^{4} & +\left(K_{a}+K_{b}+\frac{C_{B}}{1+C_{s} / C_{\mathrm{H}^{+} \text {bath }}}\right) C_{\mathrm{H}^{+}}^{3} \\
& +\left(K_{a} K_{b}-C_{\mathrm{H}^{+} \text {bath }}^{2}+\frac{K_{a}\left(C_{B}-C_{A}\right)}{1+C_{s} / C_{\mathrm{H}^{+} \text {bath }}}\right) C_{\mathrm{H}^{+}}^{2} \\
& -\left(\left(K_{a}+K_{b}\right) C_{\mathrm{H}^{+} \text {bath }}^{2}+\frac{K_{a} K_{b} C_{A}}{1+C_{s} / C_{\mathrm{H}^{+} \text {bath }}}\right) C_{\mathrm{H}^{+}} \\
& -K_{a} K_{b} C_{\mathrm{H}^{+} \text {bath }}^{2}=0,
\end{aligned}
$$

where $C_{A}=C_{A H}+C_{A^{-}}$and $C_{B}=C_{B \mathrm{OH}}+C_{B^{+}}$represent the total concentration of acidic and basic groups, respectively.

\section{Kinetic response}

The nonequilibrium swelling response to changes in bath composition can be influenced by many competing rate processes. The mechanical, or poroelastic, response time $e^{20,21}$ involves the elastic reconfiguration of the network and the simultaneous fluid flow into or out of the network. During the swelling process the redistribution of ions in the electrical double layers take a few nanoseconds to complete. The linearized diffusion of ions within the hydrogel matrix has an associated time constant $\tau_{d} \sim l^{2} / D_{i}$, where $l$ represents the hydrogel dimension and $D_{i}$ the ion diffusion coefficient. Normally, ion diffusion processes take place on a much shorter time scale than the elastic reconfiguration of the network. If ion binding interactions take place, however, the ion diffusion time must be modified. The effect of hydrogen ion binding to acidic groups, for example, is to slow the diffusion process. ${ }^{22}$ The effective diffusion coefficient in this case can be written as 


$$
D_{i, \mathrm{eff}}=\frac{D_{\mathrm{H}^{+}}}{1+\frac{C_{A} K_{a}}{\left(K_{a}+C_{\mathrm{H}^{+}}\right)^{2}}},
$$

where $C_{A}$ is the total binding site concentration. For small perturbations from equilibrium with a large number of free binding sites the effective diffusion coefficient can be much lower than the free diffusion coefficient.

\section{EXPERIMENTAL METHODS AND PROCEDURE}

We have synthesized copolymer hydrogels of varying proportions of acryl-amido methyl propyl sulfonic acid (AMPS-H) and methacryl-amido propyl trimethyl ammonium chloride (MAPTA-Cl) as negative and positive monomeric species respectively. Two sets of hydrogels were made in this study. In both cases the balance point was determined using a precipitation reaction. Silver AMPS (AMPS-Ag) is initially formed from AMPS-H and $\mathrm{Ag}_{2} \mathrm{CO}_{3}$ and then mixed with MAPTA-Cl to precipitate the $\mathrm{Ag}$ and $\mathrm{Cl}$ counter ions. The first set of hydrogels were made at a total concentration of $700 \mathrm{mM}$ and had gelation diameters of $505 \mu \mathrm{m}$. In the second set of hydrogels, intended for the kinetic study, the total concentration was $1.4 \mathrm{M}$ and the gelation diameters were $205 \mu \mathrm{m}$.

\section{A. Sample preparation}

The AMPS-MAPTA polyampholyte pregel solution was prepared by initially dissolving 0.2 moles of AMPS-H in 200 $\mathrm{mL}$ of $\mathrm{H}_{2} \mathrm{O}$. Throughout the procedure the solution was kept cool using an ice bath in order to avoid polymerization. While stirring the hydrogen AMPS solution 0.1 moles of $\mathrm{Ag}_{2} \mathrm{CO}_{3}$ was slowly added to produce $\mathrm{CO}_{2}$ and AMPS-Ag. The solution was then centrifuged at $3000 \mathrm{rpm}$ and filtered with a $0.2 \mu \mathrm{m}$ filter. After adding MAPTA-Cl the resulting $\mathrm{AgCl}$ precipitate was filtered out using a $0.2 \mu \mathrm{m}$ filter. Small aliquots of the AMPS-MAPTA solution were tested with MAPTA-Cl and AMPS-Ag to ensure the solution had an equal concentration of AMPS and MAPTA monomers. The balanced stock solution was then diluted to the required concentrations.

Hydrogels were made using $8.6 \mathrm{mM} \mathrm{N}, \mathrm{N}^{\prime}$-methylenebis-acrylamide (crosslinker) and $1.76 \mathrm{mM}$ ammonium persulfate (initiator) at a gelation temperature of $60{ }^{\circ} \mathrm{C}$. Hydrogel samples with a charge offset were made by adding excess AMPS-H or MAPTA-Cl while keeping the total monomer concentration at $700 \mathrm{mM}$ or $1.4 \mathrm{M}$. In the first set of hydrogels, made at $700 \mathrm{mM}$, the AMPS-H solution used to offset the balanced pregel solution was neutralized with an equal molar concentration of $\mathrm{NaOH}$. In the second set of hydrogels, made at $1.4 \mathrm{M}$, the net charge was offset by $-16 \mathrm{mM}$ by adding the appropriate amount of AMPS-H. One sample was neutralized to $p \mathrm{H} 7$ with $\mathrm{NaOH}$ and the other was left at $\approx p \mathrm{H} 2$.

\section{B. Experimental outline}

The hydrogels were removed from their pipettes and placed into individual glass cell holders. The hydrogels were

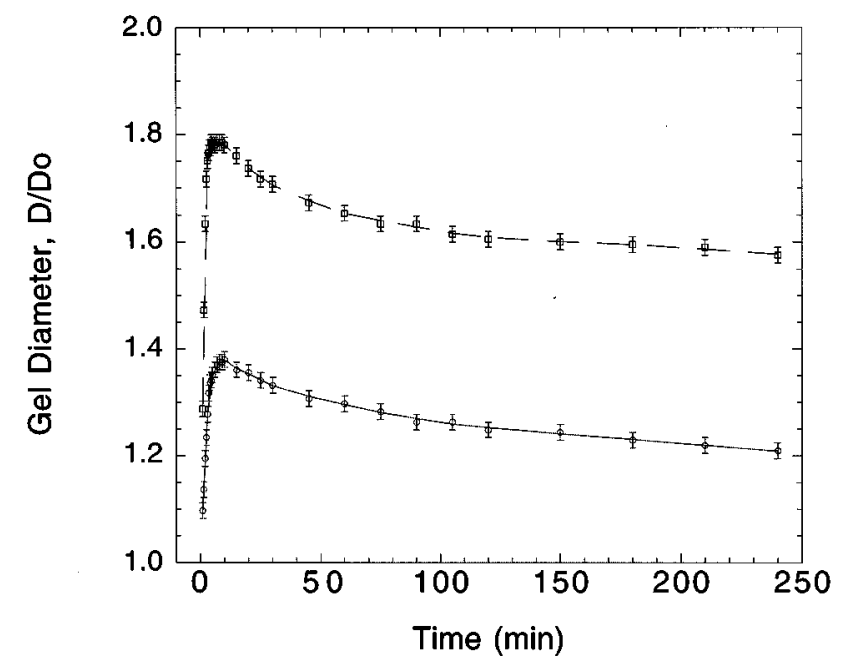

FIG. 1. Upon washing with distilled deionized water immediately after gelation a biphasic swelling response is observed. Hydrogels made at a low $p H$ (lower solid curve) do not swell to the same initial extent as those made at neutral $\mathrm{pH}$ (upper dashed curve).

then washed continuously and in parallel from the same stock solution to make quantitative comparisons. The slow kinetic response of these hydrogels make it necessary to consider time as a variable during all measurements. Hydrogel diameter measurements were made using a microscope equipped with a charged coupled device camera. Swelling diameter error estimates were based on the video resolution.

\section{RESULTS AND DISCUSSION}

Figure 1 shows the initial swelling pattern of hydrogels prepared at $p \mathrm{H} 2$ and $p \mathrm{H} 7$ upon washing with deionized distilled water. The initial transient response of both hydrogels lasts approximately five to ten minutes and appears to be dominated by the matrix poroelastic response. The lower swelling peak value of the hydrogel made at $p \mathrm{H} 2$ may be the result of localized hydrogen ion binding to the AMPS monomers. Since these hydrogels reswell upon washing in a 0.1 $\mathrm{mM} \mathrm{NaCl}$ bath the slow reptation and loss of charged oligomers cannot be attributed to the slow collapse following the poroelastic response. Reswelling following a salt wash at 0.1 $\mathrm{mM}$ is more consistent with an ion exchange mechanism leading to the replacement of hydrogen ions by sodium ions. However, the fact that the hydrogel made at $p \mathrm{H} 2$ with no sodium ions to begin with shows an initial biphasic swelling transient strongly suggests that more complicated processes besides sodium ion exchange are involved in the initial nonequilibrium response. Furthermore, diameter measurements alone do not represent the nonuniform evolution of the hydrogel density as it swells and collapses.

Figure 2 shows the normalized hydrogel diameter as a function of charge offset after 12 hours of washing with distilled deionized water. The positive offset about the balance point in the initial swelling pattern may be attributed to error in the pregelation monomer concentrations or to other physico-chemical differences between monomers. Figure 3 


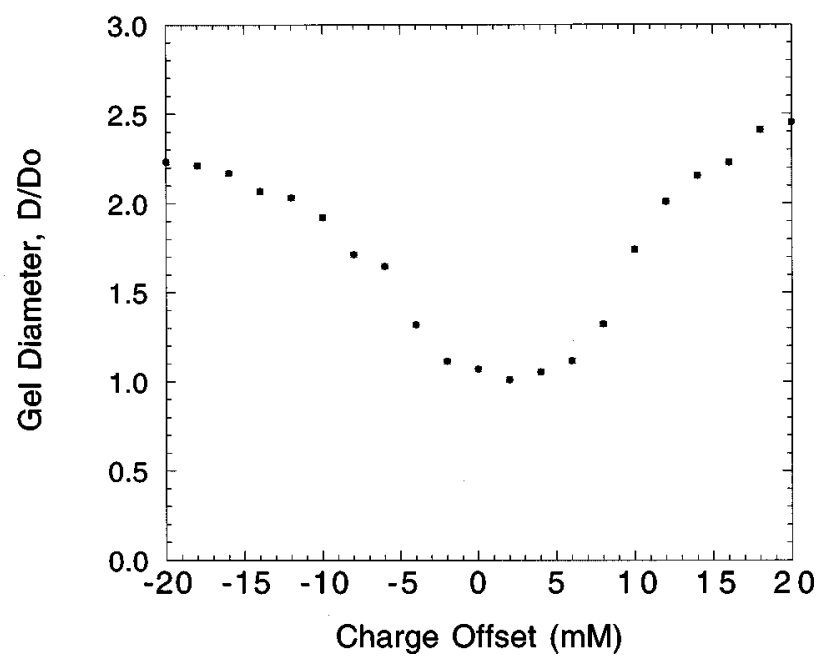

FIG. 2. Hydrogels initially swell to a symmetric distribution about the balance point within several hours of washing with distilled deionized water. The actual balance point appears to be at $+2 \mathrm{mM}$ due to experimental error or physico-chemical differences between monomers.

shows the flat skewed swelling pattern that resulted after several days of continuous washing.

Figures 4 and 5 show the results of washing negatively and positively charged hydrogels with increasing concentrations of sodium chloride. Polyampholytes near the balance point begin to swell at about $10 \mathrm{mM} \mathrm{NaCl}$. Unbalanced polyampholytes initially swell up to about $0.1 \mathrm{mM}$. This initial swelling is far more pronounced for hydrogels with a negative charge offset. The discrepancy in the peak values between the positive and negative sets of hydrogels may be partially attributed to the experimental uncertainty in the actual balance point. Above $0.1 \mathrm{mM}$ unbalanced polyampholytic hydrogels show an initial collapse followed by reswelling at $10 \mathrm{mM}$. The collapsing trend between 0.1 and 10

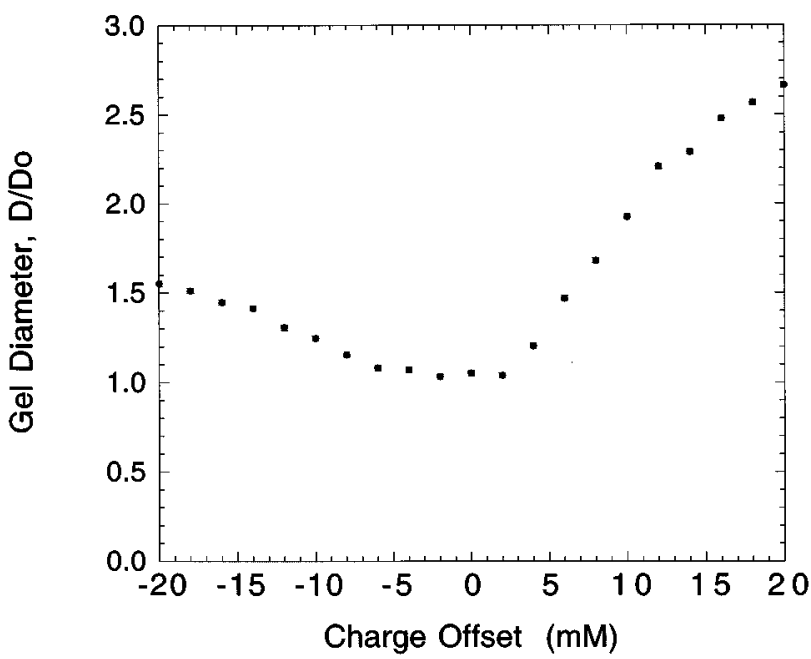

FIG. 3. Washing over a period of several days with distilled deionized water produces a skewed flattened diameter distribution.

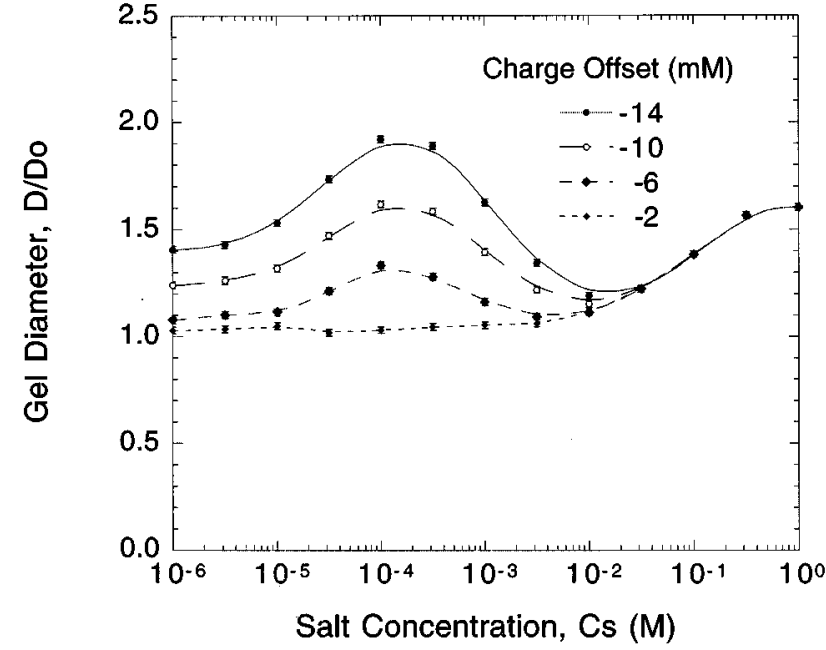

FIG. 4. Hydrogels with increasing negative charge offsets of -2 (lower), $-6,-10$, and $-14 \mathrm{mM}$ (upper) show the development of three distinct diameter swelling regimes as a function of bath electrolyte concentration. At very low bath salt concentrations the hydrogels collapse. The smooth curves are drawn to guide the eye.

$\mathrm{mM}$ represents the screen of polyelectrolyte contribution while subsequent swelling is due to the polyampholyte effect.

During the salt wash experiments the equilibration time for salt concentration changes below $0.1 \mathrm{mM}$ was several hours to days. Much faster equilibration times were observed for changes in bath salt concentrations above $0.1 \mathrm{mM}$. The slow kinetics at low sodium chloride concentrations may simply be an experimental artifact resulting from diffusion boundary layers at the hydrogel boundaries and/or the low sodium chloride availability in the wash. Diffusion reaction

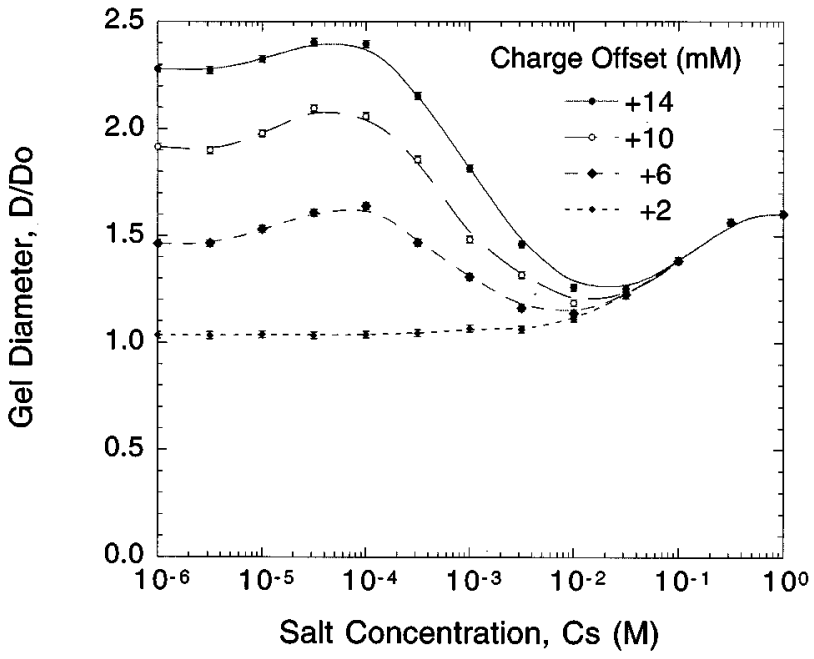

FIG. 5. Hydrogels with positive charge offsets of +2 (lower) $,+6,+10$, and $+14 \mathrm{mM}$ (upper) show similar swelling patterns as the negatively charged hydrogels with the exception of the low salt concentration regime. At low salt concentrations some collapsing is observed but not to the same extent as those of the negatively charged hydrogels. The smooth curves are drawn to guide the eye. 
kinetics or other forms of slow ion transport that are dominant at low ionic strengths may be involved. Although some caution should be used when extrapolating free monomer $p K$ values to those following gelation, a diffusion reaction mechanism based on the free monomer $p K$ values does not predict the very long observed equilibration times.

A theoretical interpretation of our experimental results requires parameter estimates for $\phi_{0}, N_{x}, \chi, \bar{v}_{\text {site }}$, and $A$. Although these parameters are not known precisely, the range of realistic values is well defined for all of them. We estimate these parameters from the hydrogel preparation conditions, the observed swelling ratios under limiting conditions and published values. The swelling ratio is then calculated as a function of bath electrolyte concentration.

Based on the pregel monomer concentrations, $C_{A 0}+C_{B 0}=700 \mathrm{mM}$, and the estimated monomer molar volume, $0.2 \mathrm{M}^{-1}$, the reference state polymer volume fraction is approximately $\phi_{0}=0.7 \mathrm{M} \times 0.2 \mathrm{M}^{-1}=0.14$. If all of the cross-linker molecules were incorporated into the hydrogel the number of monomers between cross-links, $N_{x}$, would be $N_{x}>700 \mathrm{mM} /(2 \times 8.6 \mathrm{mM}) \approx 40$. Not all the cross-linker molecules, however, lead to effective cross-links and some are not incorporated into the polymer network. As a result, a much higher value of $N_{x}$ can be anticipated.

In this experiment, the copolymer charged monomers have a high affinity for the solvent water molecules. ${ }^{10}$ The hydrophilic properties of fixed charge groups in ionexchange materials are well documented. ${ }^{25,26}$ Since the Flory interaction parameter, $\chi$, is less than 0.5 for good solvents ${ }^{23}$ our value of $\chi$ should fall in this range.

The molar value of the lattice sites in the Flory-Huggins theory can be considered an effective value ranging between that of the solvent molecules, $\bar{v}_{w} \approx 0.018 \mathrm{M}^{-1}$, and that of the monomers, $\bar{v}_{m} \approx 0.2 \mathrm{M}^{-1}$. In addition, we know that the Coulombic free energy constant, $A$, should be of the order of unity. In the case of perfectly ordered ionic crystals, ${ }^{16}$ this constant has a value close to 1.5 . Since the charge distribution in polyampholytes is much more disordered, a smaller value of $A$ should be expected.

The actual values used in the calculations presented below are $\phi_{0}=0.14, N_{x}=300, \chi=0.3, \bar{v}_{\text {site }}=0.2 \mathrm{M}^{-1}$, and $A$ $=0.4$. Figure 6 shows the swelling behavior predicted by our theory for a balanced polyampholyte (lower curve) and an unbalanced polyampholyte with a charge offset of $14 \mathrm{mM}$ (in the reference state) of either AMPS-Na (lower curve) or MAPTA-Cl (upper curve). The $p K$ values used in Fig. 6 are $p K_{a}=1.5$ and $p K_{b}=11.5$. The $p K_{a}$ was estimated from direct measurements of free monomer solutions of AMPS-H while the $p K_{b}$ was chosen to account for the observed swelling behavior.

As we proceed from concentrated to dilute electrolyte concentrations, the different contributions to the hydrogel osmotic pressure vary. For $C_{s} \approx 1 \mathrm{M}$, the Coulombic interactions between fixed ions are effectively screened and the ideal contribution to the hydrogel osmotic pressure balances the bath osmotic pressure. As a result, the equilibrium swelling is determined only by the hydrogel elastic and solvent properties and hence values of $\phi_{0}, N_{x}$, and $\chi$.

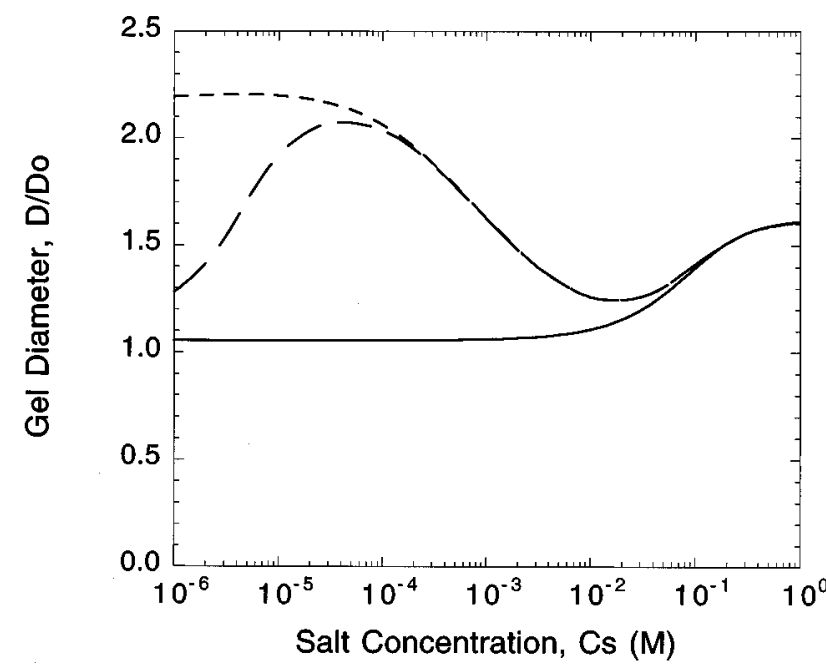

FIG. 6. Theoretical predictions for for charge offsets of 0 (lower solid), -14 (middle dashed), and $+14 \mathrm{mM}$ (upper dashed), have the same qualitative shape as those shown in Figs. 4 and 5.

Decreasing $C_{s}$ from $1 \mathrm{M}$ to $10 \mathrm{mM}$ results in the collapse of the hydrogel due to increasing fixed charge Coulombic interactions. When $C_{s}$ reaches $10 \mathrm{mM}$ the mobile ions within the hydrogel can no longer screen the fixed charges. Balanced polyampholytes, therefore, remain collapsed when the bath salt concentration is further reduced below $10 \mathrm{mM}$. Unbalanced polyampholytes, however, begin to swell due to the increasing counter ion ideal gas pressure. In unbalanced polyampholytes the combination of these two effects produces a clear minimum at $C_{s} \approx 20 \mathrm{mM}$.

As $C_{s}$ is reduced from $10 \mathrm{mM}$ unbalanced polyampholytes continue to swell until $C_{s}$ reaches $\approx 0.1 \mathrm{mM}$. Figure 6 shows that different swelling patterns are observed between unbalanced polyampholytes with excess acidic and excess basic groups when $C_{s}$ is reduced below $0.1 \mathrm{mM}$. For polyampholytes with excess sulfonic acid groups the internal concentrations of the mobile positive ions, sodium and hydrogen, are higher than that of the bath. As a result, the internal $p \mathrm{H}$ is smaller than the bath $p \mathrm{H} \approx 5.8$.

At $C_{s} \approx 0.1 \mathrm{mM}$, the actual fixed charge concentration can be estimated as $14 \mathrm{mM} / 2.1^{3} \approx 1.5 \mathrm{mM}$. Based on Donnan equilibrium, the internal and external $p \mathrm{H}$ differ by $\log (1.5$ $\mathrm{mM} / 0.1 \mathrm{mM}) \approx 1.2 p \mathrm{H}$ units. Although this internal $p \mathrm{H}$ is not low enough to affect the sulfonic acid dissociation equilibrium, the situation changes for lower electrolyte concentrations. When $C_{s} \approx 0.01 \mathrm{mM}$, for example, the internal $p \mathrm{H}$ is $5.8-\log (1.5 \mathrm{mM} / 0.01 \mathrm{mM}) \approx 3.6$. The sulfonic acid ionization degree in this case is $\alpha_{a}=1 /\left(1+C_{\mathrm{H}^{+}} / K_{a}\right) \approx 0.9925$. As a result, $0.75 \%$ of the $357 \mathrm{mM}$ of sulfonic groups, or $3 \mathrm{mM}$, are not dissociated. The net charge concentration decreases from $14 \mathrm{mM}$ to $11 \mathrm{mM}$ and the hydrogel collapses. These concentrations are given as their equivalent in the reference state for the sake of clarity.

The swelling ratio, $D / D_{0}$, continues decreasing from 10 $\mu \mathrm{M}$ to $1 \mu \mathrm{M}$ and reaches a constant value of approximately 1.2 at $1 \mu \mathrm{M}$. When $C_{s} \rightarrow 0$ local electroneutrality yields $C_{A^{-}}=C_{\mathrm{H}^{+}}+C_{B^{+}}$and the fixed charge concentration is com- 


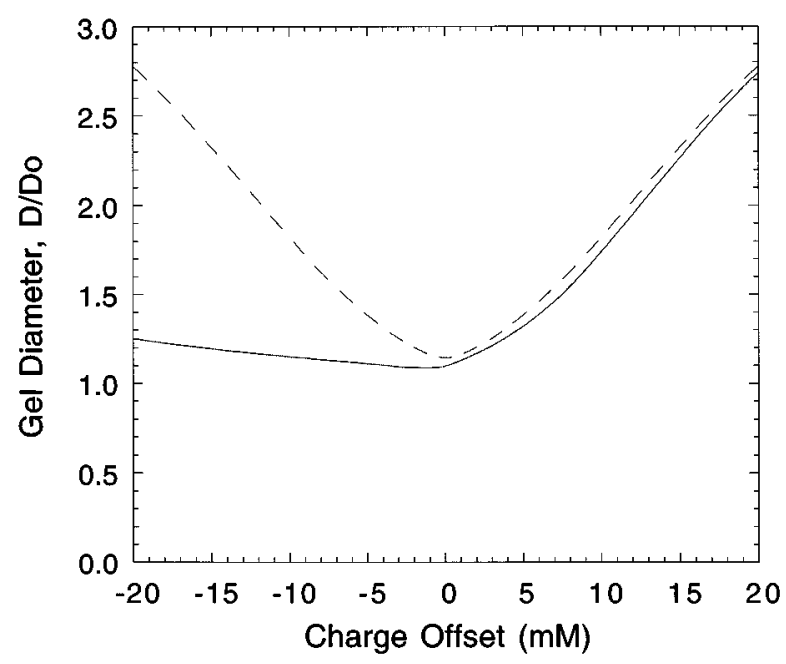

FIG. 7. Theoretical predictions also show the development of asymmetric swelling when dissociation equilibria are included in agreement with the data shown in Figs. 2 and 3.

pensated by hydrogen ions. Assuming the base groups are completely dissociated and the acid dissociation is described by Eq. (16), or the appropriate limit of Eq. (20), the minimum internal $p \mathrm{H}$ is 2.96 . This corresponds to an actual fixed charge concentration of only $1.11 \mathrm{mM}$ and an acid group ionization degree of $\alpha_{a} \approx 0.966$. Hence, when $C_{s}$ decreases below the bath hydrogen ion concentration, $1.6 \mu \mathrm{M}$, all the contributions to the hydrogel osmotic pressure reach a constant value and are dominated by the mixing and Coulombic terms.

The upper curve of Fig. 6 shows that the polyampholyte swelling with excess base groups is essentially the same as the acidic polyampholyte except at low bath ionic strengths. As $C_{s} \rightarrow 0$ the basic polyampholyte fixed charges are now compensated with bicarbonate ions. In this case, local electroneutrality, $C_{A^{-}}+C_{\mathrm{HCO}_{3}^{-}}=C_{B^{+}}$, leads to a limiting internal $p \mathrm{H}$ value of 8.72 , a fixed charge concentration of 1.26 $\mathrm{mM}$ and a basic group ionization degree of $\alpha_{b} \approx 0.988$. The higher ionization degree and fixed charge concentration explains why this hydrogel is more swollen than that with excess acidic groups.

The lower skewed curve in Fig. 7 shows the predicted swelling ratio as a function of net fixed charge concentration when the polyampholyte hydrogel is immersed in distilled water. If complete ionization is assumed, theory predicts the upper symmetrical distribution shown in Fig. 7. Asymmetry results when acid and base dissociation equilibria are included. The observed asymmetry can be attributed to the differences in monomer $p K$ values with respect to the slightly acidic external $p \mathrm{H}$. Since $(5.8-1.5)<(11.5-5.8)$ the acid group ion association is greater despite the very low $p K_{a}$.

Figure 8 shows the theoretical predictions for the swelling ratio when complete ionization of both acid and base groups is assumed over the whole electrolyte concentration range. The hydrogel parameters used are the same as those

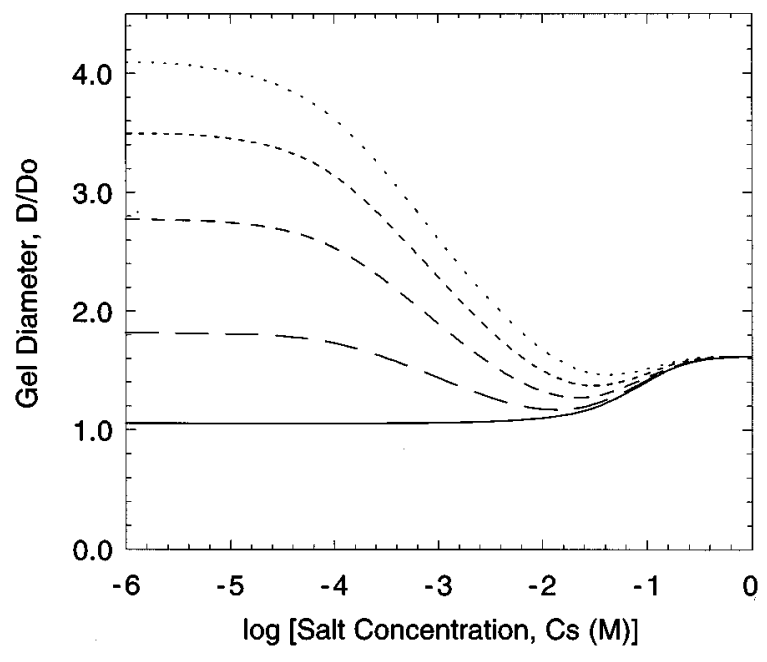

FIG. 8. If the acid base dissociation equilibria are ignored then theory shows no collapse transition at low ionic strengths. The curves correspond to charge offsets of 0 (lower curve), 10, 20,30, and $40 \mathrm{mM}$ (upper curve).

for Fig. 6, with net fixed charge concentrations of $\left|C_{B 0}-C_{A 0}\right|=0,10,20,30$, and $40 \mathrm{mM}$. Experimentally, the assumption of complete ionization may correspond to measurements carried out at relatively short times, exceeding the poroelastic response time, when electrochemical equilibrium of hydrogen and hydroxide ions between the bath and the free and bound states within the hydrogel have not been attained.

Despite the qualitative agreement with experiment, our model has a number of limitations. Experimental studies, ${ }^{8,26}$ for example, have shown that hydrogel swelling is influenced by the nature of the counter ion species and may be related to a specific counter ion condensation phenomenon. The only counter ion characteristic we have allowed in our model is the counter ion charge number which enters the ideal and Coulombic terms. Contrary to experiment, the model in its present form, cannot differentiate between lithium and sodium ions.

Recent experimental results ${ }^{10}$ have also shown that specific interactions between the mobile ions and the neutral polymer network lead to an increase in the swelling ratio with increasing $C_{s}$. In our model we have assumed that this increase is due exclusively to the screening of the attractive interactions between fixed charges. Furthermore, it is well known that the screened Coulomb potential, and the ideal contribution to the hydrogel free energy are not valid at high concentrations. This implies that a significant range of our experimental concentrations may be well beyond the limit of our theory. ${ }^{23,27}$

The use of a thermally smeared out quasilattice model for the Coulombic interactions between fixed charges in the random copolymer, instead of the Debye-Hückel theory, may be justified for the case of collapsed balanced polyampholytes. For highly swollen slightly unbalanced hydrogels, where the model can be questioned, the Coulombic term becomes less important. Since the quasilattice model tempera- 
ture dependence is different from the Debye-Hückel theory an experimental study of balanced polyampholyte swelling at different temperatures could be useful to establish the conditions for the validity of each model.

\section{CONCLUSIONS}

The combination of equilibrium and kinetic measurements in this study underscore the importance of counter ions in establishing equilibrium in polyampholyte hydrogels. Despite the very low $p K_{a}$ of AMPS-H, the flattening of the swelling distribution shown in Fig. 3 appears to be the result of hydrogen ion association to AMPS. Without added base the concentration of free bicarbonate ions is approximately 250 times the hydroxide ion concentration. As a result, most MAPTA remains in its ionized form and hydrogels containing excess MAPTA are swollen at low ionic strengths. This suggests that in practice, dissociation equilibria rather than charge offset alone determines polyampholytic equilibrium swelling at low ionic strengths.

We have shown that an extension of the Flory-Huggins theory incorporating a quasi-lattice screened Coulombic term for the hydrogel fixed charge groups can explain the observed trends for balanced and slightly unbalanced polyampholytic hydrogels. Although a high degree of parameter uncertainty exists in this simplified model, satisfactory agreement between theory and experiment has been obtained. In particular, the polyampholyte effect is predicted to be effective within a certain range of concentrations and negligible when $C_{S} \geqslant\left|C_{B^{+}}-C_{A^{-}}\right|$, in qualitative agreement with Higgs and Joanny's scaling theory. In our model the Debye screening length $1 / \kappa$ decreases with increasing $C_{s}$ until it eventually reaches values smaller than the average distance $r$ between fixed charges. As a result, the screening factor suppresses attractive electrostatic interactions between unlike fixed charges and the hydrogel swells.

\section{ACKNOWLEDGMENTS}

This work was partially supported by National Science Foundation Grant DMR 90-22933. A.E.E. acknowledges fel- lowship support from Raytheon and Rhône-Poulenc Rorer. S.M. and J.A.M. acknowledge the Consellería de Educació i Ciència (Generalitat Valenciana) and the Universitat de València for financial support received. S.M. and J.A.M. also thank the DGICYT, Ministerio de Educación y Ciencia of Spain. A.Y.G. acknowledges the support of a Kao Fellowship. We wish to thank Professor Satoru Masamune for helpful suggestions in our organic synthesis.

${ }^{1}$ Y. Kantor, H. Li, and M. K. Kardar, Phys. Rev. Lett. 69, 61 (1992).

${ }^{2}$ Y. Kantor, M. K. Kardar, and H. Li, Phys. Rev. E 49, 1383 (1994).

${ }^{3}$ Y. Kantor and M. K. Kardar, Phys. Rev. E 51, 1299 (1995).

${ }^{4}$ Y. Kantor and M. K. Kardar, Phys. Rev. E 52, 835 (1995).

${ }^{5}$ E. I. Shakhnovich and A. M. Gutin Phys. Rev. E 50, 3322 (1994).

${ }^{6}$ A. V. Dobrynin and M. Rubinstein, J. Phys. II 5, 677 (1995).

${ }^{7}$ V. Borue and I. Erukhimovich, Macromolecules 21, 3240 (1988).

${ }^{8}$ J. Corpart and F. Candau, Macromolecules 26, 1333 (1993).

${ }^{9}$ M. Skouri, J. P. Munch, S. J. Candau, S. Neyret, and F. Candau, Macromolecules 27, 69 (1994).

${ }^{10}$ J. P. Baker, H. W. Blanch, and J. M. Prausnitz, Polymer 36, 1061 (1995).

${ }^{11}$ P. G. Higgs and J. F. Joanny, J. Chem. Phys. 94, 1543 (1991).

${ }^{12}$ X. Yu, Ph.D. thesis, Massachusetts Institute of Technology, 1993.

${ }^{13}$ A. English, X. Yu, and T. Tanaka, Abstract, Materials Research Society, N3.58, 1995.

${ }^{14}$ P. J. Flory, Principles of Polymer Chemistry (Cornell University Press, Ithaca, New York, 1953).

${ }^{15}$ P. J. Flory, Polymer 20, 1317 (1979).

${ }^{16}$ C. Kittel, Introduction to Solid State Physics (Wiley, New York, 1986).

${ }^{17}$ J. P. Valleau, Chem. Phys. 129, 163 (1989).

${ }^{18}$ M. Nagasawa and S. A. Rice, Polyelectrolyte Solutions (Academic, London, 1961).

${ }^{19}$ A. Grosberg and A. Khokholov, Statistical Physics of Macromolecules (AIP Press, New York, 1994), Chap. 5.

${ }^{20}$ M. Biot, J. Appl. Phys. 12, 155 (1941).

${ }^{21}$ T. Tanaka and D. J. Fillmore, J. Chem. Phys. 70, 1214 (1979).

${ }^{22}$ J. H. Nussbaum and A. Grodzinsky, J. Membrane Sci. 8, 193 (1981).

${ }^{23}$ T. L. Hill, Introduction to Statistical Thermodynamics (Dover, New York, 1960).

${ }^{24}$ F. Helfferich, Ion Exchange (McGraw-Hill, New York, 1962).

${ }^{25} \mathrm{P}$. Simon, Ion Exchange Training Manual (Van Nostrand Reinhold, New York, 1991).

${ }^{26}$ X. Liu, Z. Tong, and O. Hu, Macromolecules 28, 3813 (1995).

${ }^{27}$ D. A. McQuarrie, Statistical Mechanics (Harper and Row, New York, 1976). 